\title{
Flow-mediated vasodilatation in the patients with anorexia nervosa
}

\author{
Palova S, Charvat J, Chlumsky J \\ Department of Medicine, 2nd Medical Faculty, Charles University and Faculty Hospital Motol, Prague, Czech Republic. \\ jiri.charvat@Ifmotol.cuni.cz
}

\begin{abstract}
Objective: To compare flow-mediated vasodilatation in patients with anorexia nervosa and healthy subjects.

Background: Endothelial dysfunction is present in the patients with anorexia nervosa. However, flow-mediated vasodilatation in the patients with anorexia nervosa in comparison with control subjects has not been yet evaluated. Methods: Flow-mediated vasodilatation in the brachial artery was examined in 30 patients with anorexia nervosa admitted to metabolic care unit for realimentation and compared to 30 control subjects.

Results: The average age of the patients with mental anorexia was $25.0 \pm 5.2$ compared to $25.5 \pm 4.5$ years of the healthy control subjects (NS). BMI was in $14.0 \pm 1.7 \mathrm{~kg} / \mathrm{m}^{2}$ in patients with anorexia nervosa comparing to $20.4 \pm 1.0 \mathrm{~kg} / \mathrm{m}^{2}$ in the healthy control subjects $(p<0.001)$.

The baseline mean diameter of the right brachial artery was $0.33 \pm 0.06 \mathrm{~cm}$ in the anorexia nervosa patients and $0.35 \pm 0.05 \mathrm{~cm}$ in the control subjects (NS). The absolute increase of brachial artery size after reactive hyperemia was $0.029 \pm 0.006 \mathrm{~cm}(9 \%)$ in the anorexia nervosa patients and $0.039 \pm 0.006 \mathrm{~cm}(11 \%)$ in the control subjects $(p=0.002)$. After realimentation, the baseline mean diameter of the right brachial artery was comparable to the result before nutrition intervention $-0.34 \pm 0.05 \mathrm{~cm}$ but brachial artery increase due to reactive hyperemia was $0.036 \pm 0.05 \mathrm{~cm}(10.5 \%)$. It was for $19 \%$ higher compared to the first examination $(p<0.001)$

Conclusion: Flow-mediated vasodilatation is decreased in the patients with anorexia nervosa in comparison with the healthy control subjects and improves after realimentation (Tab. 1, Ref. 20). Full Text in PDF www.elis.sk. Key words: anorexia nervosa, flow-mediated vasodilatation, realimentation.
\end{abstract}

The natural history of anorexia nervosa is characterized by an increased cardiac mortality rate due to cardiovascular complications following the massive weight loss (1). The effect of severe caloric deprivation on heart size, myocardial mass, left ventricular function has been documented (2). The abnormalities of the heart rate variability as well as the variability of QT interval on electrocardiogram has been recognized in the previous studies (3).

However, the higher mortality rate in these patients may be due to the presence of serious arrhythmias induced by metabolic or hormonal changes that may be associated with the impairment of endothelial function (4). Flow-mediated dilatation of brachial artery is a simple examination reflecting the changes of endothelial function $(5,6)$, but it has not been studied in patients with anorexia nervosa yet. The aim of the presented report was to compare flowmediated dilatation of brachial artery in patients with anorexia nervosa after the significant loss of weight with healthy slim young women as well as to evaluate the effect of realimentation on this parameter of endothelial function.

Department of Medicine, 2nd Medical Faculty, Charles University and Faculty Hospital Motol, Prague, Czech Republic

Address for correspondence: J. Charvat, MD, PhD, Medical Department of 2nd Medical Faculty of Charles University and University Hospital Prague Moto, V Uvalu 84, CZ-150 06 Prague 5, Czech Republic.

Phone: +420.2 .603280598 , Fax: +420.2 .24434019$

\section{Patients and methods}

The patients with anorexia nervosa were admitted in Metabolic Care Unit of Faculty Hospital Prague Motol for realimentation from January 2006 to December 2009. During admission procedure, clinical examination including body weight, height, laboratory screening and flow-mediated dilatation of the brachial artery was evaluated on admission.

Afterwards, nutrition intervention was implemented preferably by enteral nutrition. In case of intolerance of enteral nutrition, parenteral nutrition was applied via PICC (peripherally inserted central catheter).

The aim was BMI increase for 10 to $15 \%$ over three weeks. A day before discharge, flow-mediate dilatation of the right brachial artery was assessed again.

The examination of flow-mediated dilatation of the right brachial artery was made also in the control group of young slim women - nurses working in the same hospital.

Endothelial function was evaluated by measuring the FMD of the brachial artery using a standardized protocol $(7,8,9)$. Brachial artery reactivity studies were performed at baseline, and after nutrition intervention in patients with AN. Subjects were required to be fasting and not use any tobacco-containing products for 8 hours before the study. Subjects were placed in a supine position in a temperature-controlled room for 10 min before imaging. A blood pressure cuff was placed on the widest part of the proximal right 
forearm. Using a 10-MHz linear array vascular ultrasound transducer was located above the elbow and scanned in longitudinal sections. After recording baseline B-mode ultrasound images of the brachial artery, the cuff was inflated to $250 \mathrm{~mm} \mathrm{Hg}$ for $5 \mathrm{~min}$ to induce reactive hyperemia. Brachial artery images were obtained 60 and 90 seconds after deflation. Studies were recorded digitally. The same reader read the baseline and follow-up studies in all subjects. The primary outcome variable was the maximum FMD (in \%), the largest percentage of change in the brachial artery diameter after reactive hyperemia relative to the baseline diameter. The absolute maximum FMD (in $\mathrm{cm}$ ), the absolute difference in brachial artery size after reactive hyperemia compared to baseline, also was reported.

In our laboratory, approximately 4 weeks apart had an interscan FMD difference of only $0.36 \%(-0.63 \%$ to $+0.82 \%$, p $=0.498)$; the median inter-reader variability was $-0.24 \%$ to $+0.12 \%$, with correlations of 0.95 to $0.99(\mathrm{p}<0.001)$.

Flow-mediated dilatation of the brachial artery was measured by scanner CC-15M71-MA, Toshiba, Otawara, Japan.

All participants (patients as well as control subjects) gave the written consent. The study followed Helsinki protocol and was accepted by local ethics commission.

Continuous parameters were evaluated as the mean \pm standard deviation (SD). The evaluation of continuous parameters between groups was performed by the paired and unpaired T-test. Values of $p<0.05$ were considered to be statistically significant. The statistical analyses were performed using the statistical software Stat graphic Centurion, version XV from Stat Point Inc (Herndon, Virginia, USA).

\section{Results}

Thirty patients with anorexia nervosa and 30 healthy young women as control subjects have entered the study. Duration of anorexia nervosa in our patients was $9 \pm 4$ years.

Comparison of basic characteristics is summarized in Table 1.

The nutritional intervention lasted for $18 \pm 2$ days. After realimentation, BMI increased to $15.8 \pm 1.0 \mathrm{~kg} / \mathrm{m}^{2}(\mathrm{p}<0.001)$

The baseline mean diameter of the right brachial artery was $0.33 \pm 0.06 \mathrm{~cm}$ in anorexia nervosa patients and $0.35 \pm 0.05 \mathrm{~cm}$ in the control subjects (N.S.).

Tab. 1. Basic characteristic of the AN patients and control subjects.

\begin{tabular}{lccc}
\hline & $\begin{array}{c}\text { Control group } \\
(\mathrm{n}=30)\end{array}$ & $\begin{array}{c}\text { AN patients } \\
(\mathrm{n}=30)\end{array}$ & $\mathrm{p}$-value \\
\hline Age (years) & $25 \pm 5$ & $25 \pm 4$ & $\mathrm{NS}$ \\
Height $(\mathrm{cm})$ & $168 \pm 4$ & $168 \pm 5$ & $\mathrm{NS}$ \\
Weight $(\mathrm{kg})$ & $58 \pm 6$ & $38 \pm 5$ & $\mathrm{p}<0.01$ \\
BMI $(\mathrm{kg} / \mathrm{m} 2)$ & $20.2 \pm 1.8$ & $13.7 \pm 1.2$ & $\mathrm{p}<0.01$ \\
Systolic BP $(\mathrm{mmHg})$ & $126 \pm 7$ & $124 \pm 6$ & $\mathrm{NS}$ \\
Diastolic BP $(\mathrm{mm} \mathrm{Hg})$ & $74 \pm 6$ & $73 \pm 6$ & $\mathrm{NS}$ \\
Heart rate $($ beats/min) & $72 \pm 6$ & $50 \pm 5$ & $\mathrm{p}<0.01$ \\
Na (mmol/l) & $136 \pm 5$ & $134 \pm 6$ & $\mathrm{NS}$ \\
K (mmol/l) & $4.3 \pm 0.3$ & $4.1 \pm 0.3$ & $\mathrm{NS}$ \\
Plasma albumin (g/l) & $38 \pm 5.0$ & $40 \pm 4.2$ & $\mathrm{NS}$ \\
Serum creatinine (umol/l) & $74 \pm 11$ & $70 \pm 13$ & $\mathrm{NS}$ \\
\hline AN - anorexia nervosa, BMI - body mass index, $\mathrm{BP}-$ blood pressure
\end{tabular}

The absolute increase of brachial artery size after reactive hyperemia was $0.029 \pm 0.006 \mathrm{~cm}(9 \%)$ in anorexia nervosa patients and $0.039 \pm 0.006 \mathrm{~cm}(11 \%)$ in control subjects $(\mathrm{p}=0.002)$. After realimentation, the baseline mean diameter of the right brachial artery was comparable to the result before nutrition intervention $-0.34 \pm 0.05 \mathrm{~cm}$ but brachial artery increase due to reactive hyperemia was $0.036 \pm 0.05 \mathrm{~cm}(10.5 \%)$. It is for $19 \%$ higher comparing to the first examination $(\mathrm{p}<0.001)$

\section{Discussion}

Cardiac mortality is significantly increased in the patients suffering from anorexia nervosa. Despite of many hemodynamic and metabolic abnormalities described in the literature, the pathogenesis of cardiac death is not yet completely clear in anorexia nervosa patients (1).

Hypercholestrolemia, hypercortisolemia, low levels of essential fatty acids, estrogens and antioxidant vitamins are more prevalent in patients with anorexia nervosa and could lead to cardiovascular risk increase (10). On the other side, the study comparing intimomedial thickness of patients with mental anorexia and in healthy control subjects did not detect any difference (11).

Abnormalities of heart rate variability and the variability of QT interval may be responsible for the development of serious arrhythmias but according to the majority of reports they resolved with body weight gain during nutrition intervention (12).

Endothelial dysfunction is associated with cardiac events including sudden death $(13,14,15)$. In anorexia nervosa patients, there are several reasons for endothelial function impairment. Abnormalities of mineral metabolism are frequent in these individuals including magnesium depletion. In the previous report, the low concentration of magnesium in erythrocytes during chronic stress was associated with the impairment of flow-mediated vasodilatation (16).

The patients with anorexia nervosa have frequently low concentration of D vitamin. In the previous study, the deficiency of D vitamin was associated with inflammation-linked vascular endothelial dysfunction in middle-aged adults (17). In another study, the application of D vitamin improved endothelial function in patients with Type 2 diabetes mellitus and low vitamin D levels (18).

In patients with anorexia nervosa, thyroid functions may be impaired. Subclinical hypothyroidism was found to have significantly lower flow-mediated vasodilatation (19). Ghrelin, growth hormone and adinopectin lower plasma levels may contribute to the progression of endothelial dysfunction evaluated by flowmediated vasodilatation. The application of estrogen improves flow-mediated vasodilatation (20).

Flow-mediated dilatation of brachial artery brachial abnormality in anorexia nervosa patients might be also due to other metabolic or hormonal changes presented in anorexia nervosa patients.

In our study, flow-mediated vasodilatation of the brachial artery was significantly reduced in comparison with control subjects but it has improved after realimentation. According to our knowledge, this has not yet been reported. It confirms the presence of endothelial dysfunction after the significant weight loss in anorexia 
nervosa patients. This impairment is reversible and subsides after refeeding. The future study should evaluate the metabolic and hormonal changes that could be responsible for flow-mediated vasodilatation impairment in anorexia nervosa patients as well as the clinical relevance of this finding.

\section{Conclusion}

Flow-mediated vasodilatation is decreased in the patients with anorexia nervosa in comparison with healthy control subjects and improves after realimentation.

\section{References}

1. Neumarker KJ. Mortality and sudden death in anorexia nervosa. Int J Eat Disord 1997; 21: 205-212 .

2. Galletta F, Franzoni F, Cupisti A, Morelli E, Santoro G, Pentimone F. Early detection of cardiac dysfunction in patients with anorexia nervosa by tissue Doppler imaging. Int J Cardiol 2005; 101: 33-37.

3. Ishizawa T, Yoshiucchi K, Takimoto Y, Yamamoto Y, Akabayashi A. Heart rate and blood pressure variability and baroreflex sensitivity in patients with anorexia nervosa. Psychosom Med 2008; 70: 695-700.

4. Komaki G, Tamai H, Mukuta T, Kobayashi N, Mori K, Nahagawa T. Alterations in endothelium-associated proteins and serum thyroid hormone concentrations in anorexia nervosa. Br J Nutr 1992; 68: 67-75.

5. Kawano H, Yoshida T, Miyao Y et al. The relationship between endothelial function in the brachial artery and intima plus media thickening of the coronary arteries in patients with chest pain syndrome. Atherosclerosis 2007; 195: 361-366.

6. Mitchell GF, Parise H, Vita JA et al. Local shear stress and brachial flow-mediated dilatation: the Framigham Heart Study. Hypertension 2004; 44: 134-139.

7. Corretti MC, Anderson TJ, Benjamin EJ et al. Guidelines for the ultrasound assessment of endothelial-dependent flow-mediated vasodilation of the brachial artery: a report of the International Brachial Artery Reactivity Task Force. J Am Coll Cardiol 2002; 39: 257-265.

8. Deanfield J, Donald A, Ferri C et al. Endothelial function and dysfunction. Part I: methodological issues for assessment in the different vascular beds: A statement by the Working Group of Endothelin and Endothelial Factors of the European Society of Hypertesion. J Hypertens 2005; 23: 7-17.
9. Herrington DM, Fan L, Drum M et al. Brachial flow-mediated vasodilator responses in population-based research: methods, reproducibility and effects of age, gender and baseline diameter. J Cardiovasc Risk. 2001; 8: $319-328$.

10. Rigaud D, Tallonneau I, Verges B. Hypercholesterolaemia in anorexia nervosa: Frequency and changes during reffeeding. Diabet Metab 2009; 35: 57-63.

11. Birmingham CL, Lear SA, Kenyon J, Chan SY, Mancini GB, Frohlich J. Coronary atherosclerosis in anorexia nervosa. Int Eat Disord 2003; 34: 375-377.

12. Koschke M, Boettger MK, Macholds $C$ et al. Increased QT variability in patients with anorexia nervosa-an indicator for increased cardiac mortality? Int J Eat Disord 2010; 43: 743-750.

13. Lerman A, Zeiher AM. Endothelial function: cardiac events. Circulation 2005; 111: 363-368.

14. Yeboah J, Folsom AR, Burke GL et al. Predictive value of brachial flow-mediated dilation for incident cardiovascular events in a populationbased study: the Multi-Ethnic Study of Atherosclerosis. Circulation. 2009; 120: 502-509.

15. Shimbo D, Grahame-Clarke C, Miyake $Y$ et al. The association between endothelial dysfunction and cardiovascular outcomes in a population-based multi-ethnic cohort. Atherosclerosis. 2007; 192: 197-203.

16. Takase B, Akima T, Uehata A, Ohsuzu F, Kutita A. Effect of chronic stress and sleep deprivation on both flow-mediated dilation in the brachial artery and the intracellular magnesium level in human. Clin Cardiol 2004; 27: $223-227$.

17. Jablonski KL, Chonchol M, Pierce GL, Walker AE, Seais DR. 25-Hydroxyvitamin D deficiency is associated with inflammation-linked vascular endothelial dysfunction in middle-aged and older adults. Hypertension 2011; 57: 63-69.

18. Sugden JA, Davies JI, Witham MD, Morris AD, Struthers AD. Vitamin D improves endothelial function in patients with Type 2 diabetes mellitus and low vitamin D levels. Diabet Med 2008; 25: 320-325.

19. Cikim AS, Oflaz H, Ozbey $\mathbf{N}$ et al. Evaluation of endothelial function in subclinical hypothyroidim and subclinical hyperthyroidism. Thyroid 2004; 14: 605-609.

20. Korkmaz H, Onalan O. Evaluation of endothelial dysfunction: flowmediated dilation. Endothelium 2008; 15: 157-163.

Received December 12, 2011. Accepted August 18, 2013. 